\title{
TWISTED TOPOLOGICAL GRAPH ALGEBRAS
}

\author{
HUI LI
}

(Received 11 January 2015; first published online 6 March 2015)

\begin{abstract}
2010 Mathematics subject classification: primary 46L05.
Keywords and phrases: $C^{*}$-algebra, $C^{*}$-correspondence, topological graph, sheaf cohomology, Cuntz-Pimsner algebra.
\end{abstract}

The field of $C^{*}$-algebras has been influential because of its close relationship to mathematical physics and geometry and its deep classification programme. Graph algebras (see $[8,9])$ which are based on graph theory provide a large class of examples of $C^{*}$-algebras which are classifiable. The advantages of graph algebras are that the structure of a graph $C^{*}$-algebra, including the ideal structure and the $K$-theory, can be read off directly from the underlying graph.

Many authors have studied various generalisations of graph algebras. On the one hand, Kumjian and Pask in [7] constructed $k$-graph algebras which are the higher-dimensional version of graph algebras. On the other hand, Deaconu in [1] investigated topological graph algebras which are the continuous version of graph algebras associated with single local homeomorphisms of second countable locally compact Hausdorff spaces. Katsura gave a complete concept of topological graph algebras in [3-6].

Twisted $C^{*}$-algebras, which incorporate suitable cohomological data into the existing construction of $C^{*}$-algebras, provide new examples of $C^{*}$-algebras frequently exhibiting strong connections with the twisting cohomology data. The survey paper [13] provides many interesting examples and gives a detailed motivation for studying twisted $C^{*}$-algebras. For graph algebras, it seems that there are two interesting types of twisted $C^{*}$-algebras. On the one hand, Kumjian et al. in $[10,11]$ studied twisted $k$-graph algebras. On the other hand, Deaconu et al. in [2] investigated twisted groupoid $C^{*}$-algebras obtained from single local homeomorphisms.

In my $\mathrm{PhD}$ thesis, I incorporate a 1-cocycle from the sheaf cohomology group into Katsura's topological graph algebra and obtain a new $C^{*}$-algebra which is called the twisted topological graph algebra. I provide examples to demonstrate that the

Thesis submitted to the University of Wollongong in February 2014; degree approved on 7 May 2014; supervisors: David Pask and Aidan Sims.

(c) 2015 Australian Mathematical Publishing Association Inc. 0004-9727/2015 \$16.00 
twisted topological graph algebras can differ from the untwisted ones. I investigate the twisted topological graph algebra by introducing a new type of representation called a covariant twisted Toeplitz representation and showing that the Cuntz-Pimsner algebra of the twisted graph correspondence is generated by a universal covariant twisted Toeplitz representation of the graph. I expand on Katsura's ideas to prove fundamental results about the twisted topological graph algebra. In particular, I establish a version of the Cuntz-Krieger uniqueness theorem and study the ideal structure for the twisted topological graph algebra.

The construction of twisted topological graph algebras includes the twisted groupoid $C^{*}$-algebras in [2] and gives a complete twisted theory for Katsura's topological graph algebras. Another remark is that when applying the constructions of twisted $k$-graph algebras or twisted topological graph algebras to one-dimensional discrete graphs, one will only gain ordinary graph algebras.

A succinct account of the construction of twisted topological graph algebras can be found in the paper [12].

\section{References}

[1] V. Deaconu, 'Groupoids associated with endomorphisms', Trans. Amer. Math. Soc. 347 (1995), 1779-1786.

[2] V. Deaconu, A. Kumjian and P. Muhly, 'Cohomology of topological graphs and Cuntz-Pimsner algebras', J. Operator Theory 46 (2001), 251-264.

[3] T. Katsura, 'A class of $C^{*}$-algebras generalizing both graph algebras and homeomorphism $C^{*}$ algebras I. Fundamental results', Trans. Amer. Math. Soc. 356 (2004), 4287-4322.

[4] T. Katsura, 'A class of $C^{*}$-algebras generalizing both graph algebras and homeomorphism $C^{*}$-algebras II. Examples', Internat. J. Math. 17 (2006), 791-833.

[5] T. Katsura, 'A class of $C^{*}$-algebras generalizing both graph algebras and homeomorphism $C^{*}$ algebras III. Ideal structures', Ergodic Theory Dynam. Systems 26 (2006), 1805-1854.

[6] T. Katsura, 'A class of $C^{*}$-algebras generalizing both graph algebras and homeomorphism $C^{*}$-algebras. IV. Pure infiniteness', J. Funct. Anal. 254 (2008), 1161-1187.

[7] A. Kumjian and D. Pask, 'Higher rank graph $C^{*}$-algebras', New York J. Math. 6 (2000), 1-20.

[8] A. Kumjian, D. Pask and I. Raeburn, 'Cuntz-Krieger algebras of directed graphs', Pacific J. Math. 184 (1998), 161-174.

[9] A. Kumjian, D. Pask, I. Raeburn and J. Renault, 'Graphs, groupoids, and Cuntz-Krieger algebras', J. Funct. Anal. 144 (1997), 505-541.

[10] A. Kumjian, D. Pask and A. Sims, 'Homology for higher-rank graphs and twisted $C^{*}$-algebras', J. Funct. Anal. 263 (2012), 1539-1574.

[11] A. Kumjian, D. Pask and A. Sims, On twisted higher-rank graph $C^{*}$-algebras, Trans. Amer. Math. Soc., to appear, arXiv:1112.6233.

[12] H. Li, 'Twisted topological graph algebras', Houston J. Math. (2016), to appear, arXiv:1404.7756.

[13] I. Raeburn, A. Sims and D.P. Williams, 'Twisted actions and obstructions in group cohomology', in: $C^{*}$-algebras (Münster, 1999) (Springer, Berlin, 2000), 161-181.

HUI LI, Research Center for Operator Algebras,

Department of Mathematics, East China Normal University (Minhang Campus), 500 Dongchuan Road, Minhang District, Shanghai 200241, China

e-mail: hli@math.ecnu.edu.cn 